\title{
A Laborsaving, Timesaving, and More Reliable Strategy for Separation of Low-Molecular-Mass Phosphoproteins in Phos-tag Affinity Electrophoresis
}

\author{
Emiko Kinoshita-Kikuta ${ }^{1}$, Eiji Kinoshita ${ }^{1} \&$ Tohru Koike $^{1}$ \\ ${ }^{1}$ Department of Functional Molecular Science, Institute of Biomedical \& Health Sciences, Hiroshima University, \\ Hiroshima, Japan \\ Correspondence: Eiji Kinoshita, Department of Functional Molecular Science, Institute of Biomedical \& Health \\ Sciences, Hiroshima University, Hiroshima 734-8553, Japan. Tel: 81-82-257-5281. E-mail: \\ kinoeiji@hiroshima-u.ac.jp
}

Received: August 8, 2012 Accepted: September 3, 2012 Online Published: September 10, 2012

doi:10.5539/ijc.v4n5p1 URL: http://dx.doi.org/10.5539/ijc.v4n5p1

\begin{abstract}
Reversible phosphorylation is a key signaling mechanism for modulating the functional properties of proteins involved in numerous cellular events. Abnormal protein phosphorylation causes many human diseases. Experimental procedures for the determination of the phosphorylation status of certain proteins are therefore very important in relation to studies on a diverse range of physiological and pathological processes. We have previously reported a novel phosphate-affinity sodium dodecyl sulfate-polyacrylamide gel electrophoresis (SDS-PAGE) technique using a dizinc(II) complex of the phosphate-binding ligand Phos-tag in conjunction with a neutral-pH gel system to detect shifts in the mobilities of phosphoproteins ( $\mathrm{Zn}^{2+}-$ Phos-tag SDS-PAGE). However, this handmade gel-based procedure is often laborious and time-consuming to perform, and requires a skillful analyst. More recently, SuperSep Phos-tag precast gel has been developed on the basis of the $\mathrm{Zn}^{2+}-$ Phos-tag SDS-PAGE methodology. This novel ready-to-use system employs a neutral-pH gel containing $12.5 \%(\mathrm{w} / \mathrm{v})$ polyacrylamide and the immobilized $\mathrm{Zn}^{2+}$-Phos-tag $(50 \mu \mathrm{M})$, which is generally used in conjunction with a Tris-glycine-based electrophoretic running buffer. We examined the potential usage of a Tris- $N$-[2-hydroxy-1,1-bis(hydroxymethyl)ethyl]glycine (Tris-Tricine) buffer as an alternative running buffer for the SuperSep Phos-tag precast gel system in the analysis of low-molecular-mass phosphoproteins. Compared with Tris-glycine, the Tris-Tricine running buffer improved the resolution of 8.8-35 kDa phosphoproteins and phosphopeptides. We can therefore provide a laborsaving, timesaving, and more reliable strategy for separation of low-molecular-mass phosphoproteins in Phos-tag affinity electrophoresis.
\end{abstract}

Keywords: Phos-tag, precast gel, low molecular mass, phosphoprotein, phosphopeptide

\section{Introduction}

Separation of a phosphorylated protein and its nonphosphorylated counterpart by using gel-based electrophoresis can facilitate easily an understanding of the phosphorylation status. The classical Laemmli's procedure (Laemmli, 1970) for SDS-PAGE, which is widely used for protein separation on a polyacrylamide gel, is often used to identify phosphorylated proteins because phosphoproteins show different extents of electrophoretic migration compared with their nonphosphorylated counterparts. Uniform binding of SDS to a protein can be generally disrupted by the presence of negatively charged phosphate groups on the phosphorylated protein, resulting in the decrease in charge density on the phosphorylated protein compared with that on its nonphosphorylated counterpart. If there is a sufficient decrease in charge density of the phosphorylated form, the phosphoprotein will show a retarded migration and will appear at a position corresponding to a higher apparent molecular weight on the gel compared with its nonphosphorylated counterpart. This observation of a shift in mobility has sometimes been used as an index of protein phosphorylation in certain biological events; however, the shift in mobility on phosphorylation depends on protein-specific structural characteristics, and the number of phosphoproteins that can be analyzed by the conventional SDS-PAGE technique is limited.

We have previously reported a novel phosphate-affinity SDS-PAGE technique for the separation and detection of phosphorylated forms of proteins (Kinoshita, Kinoshita-Kikuta, \& Koike, 2009; Kinoshita, Kinoshita-Kikuta, 
Takiyama, \& Koike, 2006; Kinoshita-Kikuta, Aoki, Kinoshita, \& Koike, 2007). The affinity electrophoresis technique, $\mathrm{Mn}^{2+}$-Phos-tag SDS-PAGE, which uses a polyacrylamide-bound dimanganese(II) complex of the phosphate-binding ligand Phos-tag in conjunction with Laemmli's buffer system (an alkaline-pH gel system), has been widely used in determining the phosphorylation status of a wide variety of proteins. We have recently developed an improved Phos-tag SDS-PAGE technique in which a $\mathrm{Zn}^{2+}-$ Phos-tag complex (Kinoshita, Takahashi, Takeda, Shiro, \& Koike, 2004) is used in conjunction with a neutral-pH gel system (Kinoshita \& Kinoshita-Kikuta, 2011; Kinoshita, Kinoshita-Kikuta, \& Koike, 2012; Kinoshita-Kikuta, Kinoshita, \& Koike, 2012). Because of the greater affinity that exists between the phosphate groups and dizinc(II) complex of Phos-tag under conditions of neutral $\mathrm{pH}$, the $\mathrm{Zn}^{2+}-$ Phos-tag SDS-PAGE method provides significant improvements in the detection of shifts in the mobilities of phosphoproteins. We have identified two types of neutral-pH gel system for $\mathrm{Zn}^{2+}-$ Phos-tag SDS-PAGE. The first is buffered with Bis-Tris \{2-[bis(2-hydroxyethyl)amino]-2-(hydroxymethyl)propane-1,3-diol $\}$ and hydrochloric acid (Bis-Tris-HCl buffer), and the second is buffered with Tris [2-amino-2-hydroxymethyl(propane)-1,3-diol] and acetic acid (Tris-AcOH buffer). The advantages of these two systems have been demonstrated by the visualization of up-shifted bands of certain phosphoproteins that could not be detected by using the previous $\mathrm{Mn}^{2+}-\mathrm{Phos}$-tag SDS-PAGE system. Moreover, $\mathrm{Zn}^{2+}-$ Phos-tag SDS-PAGE gels cast in a neutral buffer show better long-term stability than do $\mathrm{Mn}^{2+}-$ Phos-tag gels, which have to be cast in an alkaline buffer. However, these handmade gel-based procedures are time-consuming, are laborious, and sometimes require highly skilled operators.

More recently, SuperSep Phos-tag, a novel precast gel suitable for the detection of shifts in the mobilities of phosphoproteins, has been developed. This ready-to-use product, which uses a neutral-pH gel system based on our $\mathrm{Zn}^{2+}$-Phos-tag SDS-PAGE methodology, provides long-term stability and is guaranteed to remain useable for 6 months. The precast gel is a 12.5\% (w/v) polyacrylamide gel containing immobilized $\mathrm{Zn}^{2+}-$ Phos-tag (50 $\mu \mathrm{M})$. Although no information on the neutral-pH buffer system used in the precast gel is provided, the manufacturer recommends the use of a general Laemmli's electrophoretic running buffer consisting of $25 \mathrm{mM}$ Tris, $192 \mathrm{mM}$ glycine, and $0.10 \%$ (w/v) SDS (Tris-glycine buffer). In our original gel buffer systems for $\mathrm{Zn}^{2+}$-Phos-tag SDS-PAGE using Bis-Tris-HCl and Tris-AcOH described above, we used an electrophoretic running buffer consisting of $100 \mathrm{mM}$ Tris, $100 \mathrm{mM}$ 3-morpholin-4-ylpropane-1-sulfonic acid (MOPS), $0.10 \%$ (w/v) SDS, and $5.0 \mathrm{mM}$ sodium bisulfite (Tris-MOPS buffer) with the Bis-Tris-HCl system or one consisting of $50 \mathrm{mM}$ Tris, $50 \mathrm{mM} \mathrm{N}$-[2-hydroxy-1,1-bis(hydroxymethyl)ethyl]glycine (Tricine), $0.10 \%$ (w/v) SDS, and $5.0 \mathrm{mM}$ sodium bisulfite (Tris-Tricine buffer) with the Tris-AcOH system (Kinoshita \& Kinoshita-Kikuta, 2011; Kinoshita et al., 2012; Kinoshita-Kikuta et al., 2012). In this study, we attempeted to employ these two running buffers, Tris-MOPS and Tris-Tricine, in the SuperSep Phos-tag precast gel system, and we evaluated the resolving power of the system in the separation of phosphoproteins in comparison with that achieved by using Tris-glycine running buffer.

\section{Materials and Methods}

\subsection{Materials}

SuperSep Phos-tag and SuperSep Ace precast gels are commercially available from Wako Pure Chemical Industries, Ltd. (Osaka, Japan, http://www.wako-chem.co.jp/english/labchem/journals/proteomics2012/ index.htm\#03). Bovine intestinal mucosa alkaline phosphatase (AP), bovine milk $\alpha$-casein, bovine milk $\beta$-casein, chicken egg ovalbumin, porcine pepsin, and bovine serum albumin (BSA) were all purchased from Sigma-Aldrich (St. Louis, MO, USA). Calyculin A and anti-histone H3 antibody were purchased from Cell Signaling Technology (Danvers, MA, USA). Anti-vimentin mouse monoclonal antibody (clone V9) was purchased from Santa Cruz Biotechnology (Santa Cruz, CA, USA). Cyanogen bromide (CNBr) was purchased from Nacalai Tesque (Kyoto, Japan).

\subsection{Dephosphorylation of $\beta$-Casein, $\alpha$-Casein, and Pepsin}

To examine the dephosphorylation status of $\beta$-casein, $\alpha$-casein, and pepsin that had been treated with AP, we performed a separate in vitro phosphatase assay for each phosphoprotein in a $50 \mathrm{mM}$ Tris- $\mathrm{HCl}$ buffer $(\mathrm{pH} 9.0$, $0.20 \mathrm{~mL}$ ) containing $1.0 \mathrm{mM} \mathrm{MgCl}_{2}, 50 \mu \mathrm{g}$ of the appropriate phosphoprotein, and 3.3 units $(0.80 \mathrm{ng})$ of AP at $37{ }^{\circ} \mathrm{C}$ for $0-60 \mathrm{~min}$. A $3 \times$ sample-loading dye solution $(0.10 \mathrm{~mL})$ for SDS-PAGE, consisting of $195 \mathrm{mM}$ Tris- $\mathrm{HCl}(\mathrm{pH} 6.8), 3.0 \%(\mathrm{w} / \mathrm{v})$ SDS, 15\% (v/v) 2-sulfanylethanol, 30\% (v/v) glycerol, and $0.10 \%(\mathrm{w} / \mathrm{v})$ Bromophenol Blue (BPB), was added to the reaction mixture to quench the reaction in a desired time.

\subsection{Preparation of Cell Lysate}

For preparation of a lysate from HeLa cells, the cells $\left(10^{7}\right.$ cells $)$ were incubated in a Dulbecco's modified Eagle medium (Life Technologies, Carlsbad, CA, USA) containing 10\% (v/v) fetal bovine serum, 100 units $/ \mathrm{ml}$ 
penicillin, and $100 \mu \mathrm{g} / \mathrm{ml}$ streptomycin under a humidified atmosphere of $5 \% \mathrm{CO}_{2}$ and $95 \%$ air at $37{ }^{\circ} \mathrm{C}$, and then treated with $0 \mathrm{nM}$ or $100 \mathrm{nM}$ calyculin A for $30 \mathrm{~min}$. After treatment, each culture was washed twice with a Tris-buffered saline (TBS) solution containing $10 \mathrm{mM}$ Tris- $\mathrm{HCl}(\mathrm{pH} 7.5)$ and $0.10 \mathrm{M} \mathrm{NaCl}$. The cultures were then lysed in $0.50 \mathrm{~mL}$ of a $1 \times$ sample-loading dye solution for SDS-PAGE. The lysate sample solutions were sonicated briefly, boiled for $5 \mathrm{~min}$, and stored at $-20^{\circ} \mathrm{C}$.

\subsection{SDS-PAGE}

Electrophoresis using the SuperSep Phos-tag or SuperSep Ace precast gel was performed at $20 \mathrm{~mA} / \mathrm{gel}$ and room temperature until the BPB dye, present in a sample-loading dye solution, reached the bottom of the gel. We used three electrphoretic running buffers, Tris-glycine [25 mM Tris, $192 \mathrm{mM}$ glycine, and $0.10 \%(\mathrm{w} / \mathrm{v})$ SDS], Tris-Tricine $[50 \mathrm{mM}$ Tris, $50 \mathrm{mM}$ Tricine, and $0.10 \%$ (w/v) SDS], and Tris-MOPS [100 mM Tris, $100 \mathrm{mM}$ MOPS, and $0.10 \%(w / v)$ SDS] for the SuperSep precast gel system. Gels were analyzed by gel staining with Coomassie Brilliant Blue G-250 (colloidal CBB solution) or by Western blotting.

\subsection{Western Blotting}

After electrophoresis, the SuperSep Phos-tag gels were soaked in a solution containing $25 \mathrm{mM}$ Tris, $192 \mathrm{mM}$ glycine, $10 \%(\mathrm{v} / \mathrm{v}) \mathrm{MeOH}$, and $1.0 \mathrm{mM}$ EDTA for $10 \mathrm{~min}$, and then soaked in a blotting buffer containing 25 $\mathrm{mM}$ Tris, $192 \mathrm{mM}$ glycine, and 10\% (v/v) MeOH for $10 \mathrm{~min}$. The gels were electroblotted to a PVDF membrane by using a Bio-Rad Mini Trans-Blot cell (Hercules, CA, USA). After electroblotting, the protein-blotting membrane was soaked in an aqueous solution containing $10 \mathrm{mM}$ Tris- $\mathrm{HCl}(\mathrm{pH} 7.5), 0.10 \mathrm{M} \mathrm{NaCl}$, and $0.10 \%$ (v/v) Tween 20 (TBS-T solution) for $1 \mathrm{~h}$, then blocked by treatment with a TBS-T solution containing $1.0 \%$ (w/v) BSA for $1 \mathrm{~h}$. Immunoprobings for histone H3 and vimentin were performed by using an anti-histone H3 antibody and an anti-vimentin antibody, respectively. The target proteins were detected as enhanced chemiluminescence signals by using Lumigen TMA-6 (Lumigen, Southfield, MI, USA) and an LAS 3000 image analyzer (Fujifilm, Tokyo, Japan).

\subsection{Preparation of Peptides by Digestion with Cyanogen Bromide}

Ovalbumin (1.0 mg, $22 \mathrm{nmol})$ or $\beta$-casein $(1.0 \mathrm{mg}, 42 \mathrm{nmol})$ was dissolved in $70 \%(\mathrm{v} / \mathrm{v})$ aqueous formic acid $(0.10 \mathrm{~mL}), \mathrm{CNBr}(3.0 \mathrm{mg}, 28 \mu \mathrm{mol})$ was added, and the mixture was incubated for $24 \mathrm{~h}$ at room temperature. After the incubation, the mixture was evaporated and the residue was dissolved in distilled water. Complete dephosphorylation of the digested peptides was performed by treatment with AP (3.3 units) in a buffer solution of $50 \mathrm{mM}$ Tris- $\mathrm{HCl}$ (pH 9.0) and $10 \mathrm{mM} \mathrm{MgCl}$. The $\mathrm{CNBr}$-digested ovalbumin or $\beta$-casein peptides and the corresponding AP-treated peptides were subjected to SDS-PAGE in $15 \%(\mathrm{w} / \mathrm{v})$ polyacrylamide gel and the targeted band was excised from the gel. The phosphopeptides (14.7 and $8.8 \mathrm{kDa})$ derived from ovalbumin, the phosphopeptide $(11.9 \mathrm{kDa})$ derived from $\beta$-casein, and the corresponding dephosphorylated peptides produced by AP treatment were then extracted.

\section{Results and Discussion}

\subsection{Comparison of Mobilities of Standard Phosphoproteins}

First, we measured the electrophoretic mobilities of three commercially available standard phosphoproteins, $\beta$-casein $(24 \mathrm{kDa}), \alpha$-casein $(24 \mathrm{kDa})$, and pepsin $(35 \mathrm{kDa})$, in the SuperSep Phos-tag precast gel system with Tris-glycine (T-G), Tris-Tricine (T-T), or Tris-MOPS (T-M) as the electrophoretic running buffer (Figure 1). In the cases of $\beta$-casein (Figure 1A) and $\alpha$-casein (Figure 1B), the phosphoprotein migrated a shorter distance than did its completely dephosphorylated counterpart prepared by treating the phosphoprotein with AP for $60 \mathrm{~min}$. Partially dephosphorylated forms of the proteins were observed as multiple bands. In the systems using Tris-Tricine and Tris-MOPS buffers, more phosphorylated species were detected than in the system in which Tris-glycine buffer was used. However, in the Tris-MOPS system, we found that the degree of migration of all bands was much smaller and that bands were relatively distorted. In the case of pepsin (Figure 1C), complete separation of the phosphorylated forms from their dephosphorylated counterparts was observed only in the Tris-Tricine system. In contrast, there was only a slight difference in the migration patterns of the phosphorylated and the dephosphorylated forms in the Tris-glycine and Tris-MOPS systems. These results show that the use of Tris-Tricine as the running buffer permits greater resolution in separation analyses of standard phosphoproteins than that achieved by using the Tris-glycine buffer. All completely dephosphorylated standard phosphoproteins obtained by AP treatment for 60 min were confirmed by using Pro-Q Diamond phosphoprotein gel staining (Life Technologies) (Steinberg et al., 2003) that there was no fluorescent signal in each treated protein band (data not shown). 
(A) $\beta$-casein

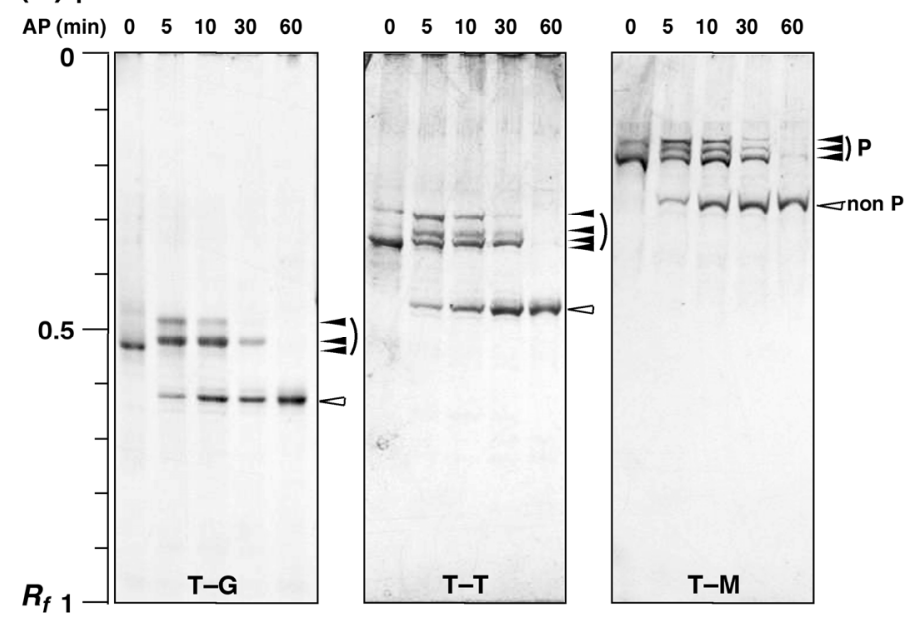

(B) $\alpha$-casein

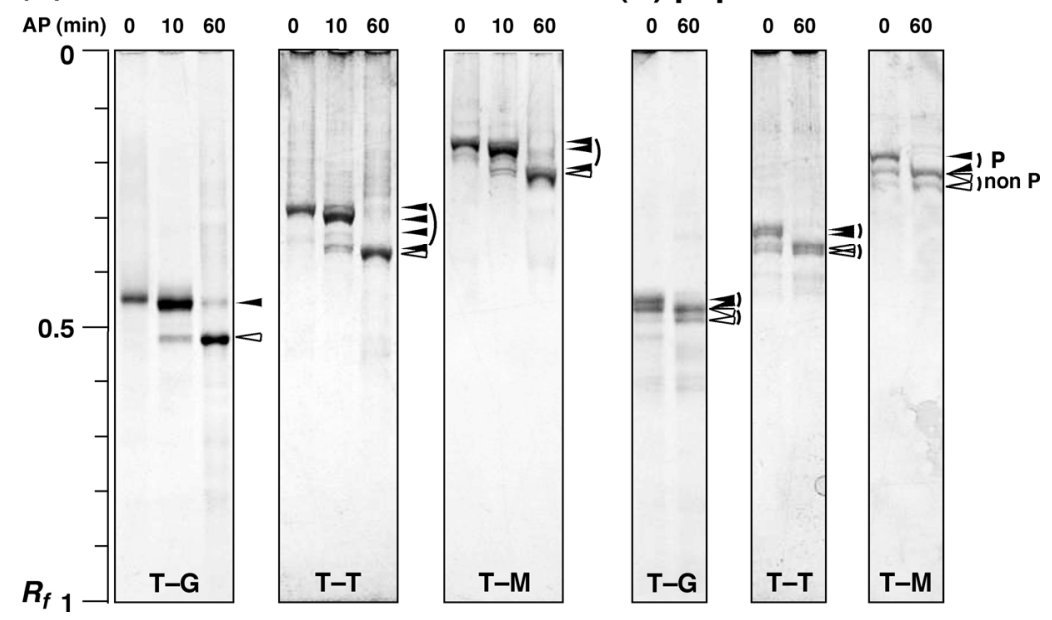

Figure 1. Comparison of mobilities of standard phosphoproteins in the SuperSep Phos-tag system with Tris-glycine (T-G), Tris-Tricine (T-T), or Tris-MOPS (T-M) running buffer. (A) $\beta$-Casein and $\beta$-casein treated with AP for 5, 10, 30, or $60 \mathrm{~min}$; (B) $\alpha$-casein or $\alpha$-casein treated with AP for 10 or $60 \mathrm{~min}$; and (C) pepsin or pepsin treated with AP for $60 \mathrm{~min}$. Each lane contains $1.0 \mu \mathrm{g}$ of protein. Left, center, and right panels represent

the SuperSep Phos-tag systems with Tris-glycine (T-G), Tris-Tricine (T-T) or Tris-MOPS (T-M) as the running buffer, respectively. The $R_{f}$ value of 1.0 is defined as the position of the BPB dye.

\subsection{Comparison of Mobilities of Intracellular Phosphoproteins}

To examine the separation power of the SuperSep Phos-tag precast gel system for phosphoproteins with a range of molecular masses, we measured and compared the mobilities of the intracellular phosphoproteins histone $\mathrm{H} 3$ $(15 \mathrm{kDa})$ and vimentin $(54 \mathrm{kDa})$ by using Tris-glycine $(\mathrm{T}-\mathrm{G})$ or Tris-Tricine $(\mathrm{T}-\mathrm{T})$ running buffer (Figure 2). These phosphoproteins were selected as targets because their molecular masses cover the range that is of interest. Sets of lysates from HeLa cells treated with $0 \mathrm{nM}$ (control) or $100 \mathrm{nM}$ calyculin A (an inhibitor of serine/threonine phosphatase) were analyzed by the SeperSep Phos-tag precast gels. And then, the presence of three phosphorylated species of histone $\mathrm{H} 3$ induced by treatment with calyculin A (right lane: + ) was confirmed (Figure 2A) by Western blotting with an anti-histone $\mathrm{H} 3$ antibody. Both banding images were very similar to that obtained with our original Bis-Tris-buffered gel system (Kinoshita et al., 2012). Phosphorylated species produced from vimentin by treatment with calyculin A were also observed as several up-shifted bands detected by immunoblotting with an anti-vimentin antibody (Figure 2B); four phosphorylated species were detected in the system using Tris-glycine and two phosphorylated species were detected in the system using Tris-Tricine. 


\section{(A) histone $\mathrm{H3}$ (15 kDa)}

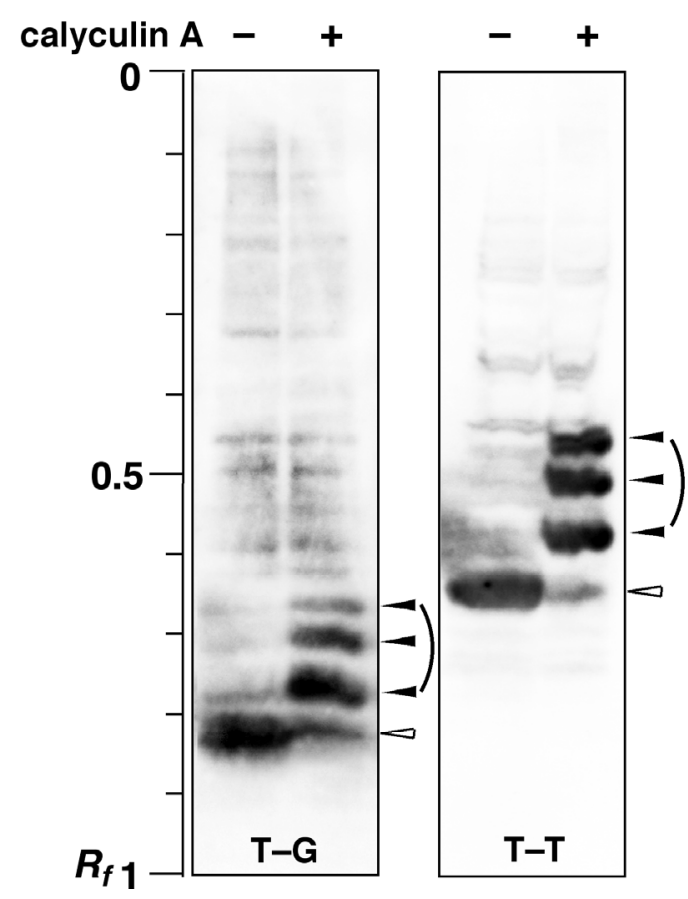

\section{(B) vimentin (54 kDa)}

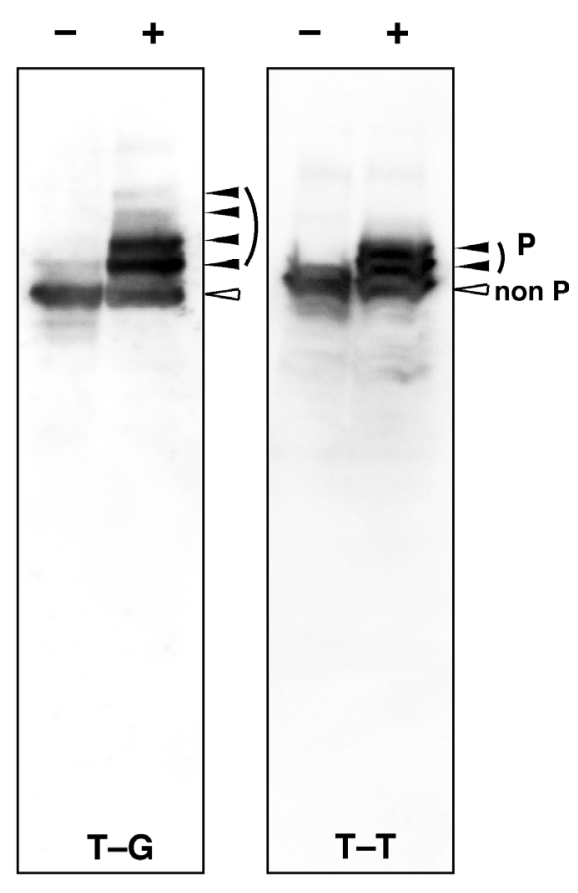

Figure 2. Comparison of mobilities of intracellular phosphoproteins in the SuperSep Phos-tag system with Tris-glycine ( $\mathrm{T}-\mathrm{G})$ or Tris-Tricine (T-T) as the running buffer. The lysates $(20 \mu \mathrm{g}$ proteins) were treated with 0 $\mathrm{nM}$ (lane: -) or $100 \mathrm{nM}$ calyculin A (lane: + ) and subjected to electrophoresis on SuperSep Phos-tag followed by immunoblotting with the anti-histone H3 antibody (A) or with the anti-vimentin antibody (B). Left and right panels represent the SuperSep Phos-tag systems with Tris-glycine (T-G) or Tris-Tricine (T-T) as the running buffer, respectively. The $R_{f}$ value of 1.0 is defined as the position of the BPB dye.

However, our original Tris-buffered gel system $\left[8.0 \%\right.$ (w/v) polyacrylamide and $25 \mu \mathrm{M} \mathrm{Zn}^{2+}$-Phos-tag] using the Tris-Tricine running buffer has permitted the resolution of eight phosphorylated forms of vimentin (Kinoshita et al., 2012). The present SuperSep Phos-tag consists of a $12.5 \%(\mathrm{w} / \mathrm{v})$ polyacrylamide gel. We therefore believe that SuperSep Phos-tag systems with polyacrylamide gels of a lower concentration are necessary to achieve maximal detail in phosphorylation analyses of proteins with molecular masses of around $50 \mathrm{kDa}$ and more. As for the analysis of relatively small phosphorotein species of histone H3, the Tris-Tricine buffer system demonstrated complete profiling of phosphorylation of the target. We also confirmed that the Western blotting procedure as a downstream application from the SuperSep Phos-tag seapartion is almost identical to that of our original $\mathrm{Zn}^{2+}$-Phos-tag SDS-PAGE methodology.

\subsection{Comparison of Mobilities of Chemical Digested Phosphopeptides}

When we used the Tris-Tricine running buffer in conjunction with the current SuperSep Phos-tag precast gel system, the histone $\mathrm{H} 3$ bands showed much slower migration than those observed in the system using the Tris-glycine running buffer (see Figure 2A). The differences in the separation characteristics of the systems with each of these two running buffers would be directly related to the differences in the $\mathrm{p} K_{\mathrm{a}}$ values of the functional groups of glycine and Tricine, which define the electrophoretic mobilities of the trailing ions (glycinate and Tricinate) relative to the mobilities of the proteins (Schägger, 2006; Schägger \& von Jagow, 1987). Anyway, this result suggests that the Tris-Tricine system is appropriate for the separation and analysis of phosphoproteins and/or phosphopeptides with molecular masses of less than $15 \mathrm{kDa}$. Therefore, we finally examined the separation analyses of phosphopeptides with molecular masses of $14.7,8.8$, and $11.9 \mathrm{kDa}$, obtained by chemical digestion of ovalbumin or of $\beta$-casein with $\mathrm{CNBr}$. The amino acid sequences of the ovalbumin and $\beta$-casein that we used and the molecular masses of the digested peptides are shown in Figures 3 and 4, respectively. 


\section{(A)}

MGSIGAASME FCFDVFKELK VHHANENIFY CPIAIMSALA MVYLGAKDST RTQINKVVRF DKLPGFGDSI EAQCGTSVNV HSSLRDILNQ ITKPNDVYSF SLASRLYAEE RYPILPEYLQ CVKELYRGGL EPINFQTAAD QARELINSWV ESQTNGIIRN VLQPSSVDSQ TAMVLVNAIV FKGLWEKAFK DEDTQAMPFR VTEQESKPVQ MMYQIGLFRV ASMASEKMKI LELPFASGTM SMLVLLPDEV SGLEQLESII NFEKLTEWTS SNVMEERKIK VYLPRMKMEE KYNLTSVLMA MGITDVFSSS ANLSGISSAE SLKISOAVHA AHAËINEAGR EVVGSAEAGV DAASVSEEFR ADHPFLFCIK HIATNAVLFF GRCVSP

(B)

$\begin{array}{cc}\text { MW (kDa) } & \text { start-end } \\ 0.44 & 37-41 \\ 0.52 & 224-228 \\ 0.65 & 2-9 \\ 1.24 & 213-223 \\ 1.26 & 229-240 \\ 1.28 & 289-299 \\ 1.51 & 275-286 \\ 1.63 & 198-211 \\ 2.71 & 174-197 \\ 3.21 & 10-36 \\ 3.59 & 243-274 \\ 8.80 & 302-386 \text { (containing phospho serine 345) } \\ 14.70 & 42-173 \text { (containing phospho serine 69) }\end{array}$

Figure 3. (A) The amino acid sequence of ovalbumin (UniProt Accession No. P01012). CNBr hydrolyzes peptide bonds at the $\mathrm{C}$-terminus of methionine residues. The $\mathrm{CNBr}$ cleavage sites are underlined. The phosphorylated serine residues at positions 69 and 345 are highlighted in bold italic letters. (B) The molecular weights $(\mathrm{MW}, \mathrm{kDa})$ and sequence ranges of the peptides formed by chemical digestion with $\mathrm{CNBr}$. The digested peptides of 14.7 and $8.8 \mathrm{kDa}$ were prepared as described above.

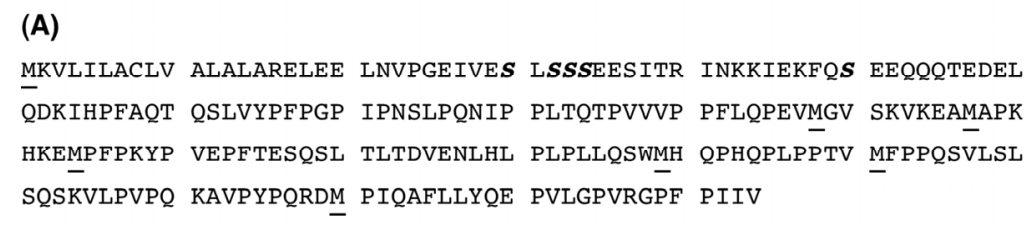

(B)

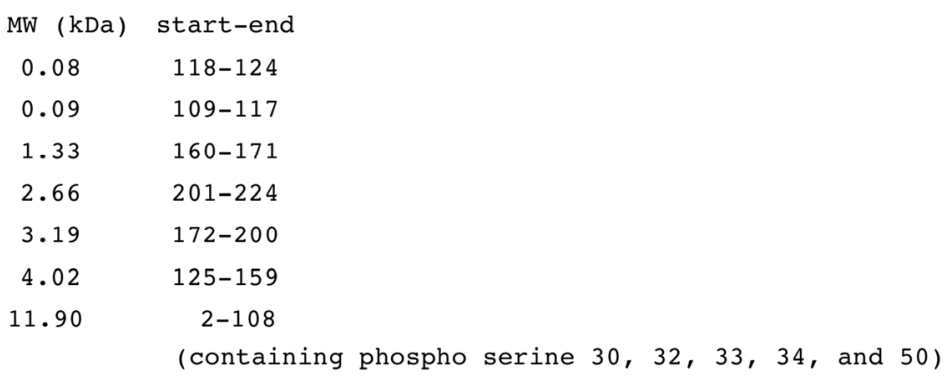

Figure 4. (A) The amino acid sequence of $\beta$-casein (UniProt Accession No. P02666). The CNBr cleavage sites are underlined. Phosphorylated serine residues at positions 30, 32, 33, 34, and 50 are highlighted as bold italic letters. (B) The molecular weights $(\mathrm{MW}, \mathrm{kDa})$ and sequence ranges of the peptides formed by chemical digestion with CNBr. The digested peptide of $11.9 \mathrm{kDa}$ was prepared as described above.

When these three phosphopeptides and their completely dephosphorylated counterparts, obtained by treatment with AP, were separated in SuperSep Ace 12.5\% (w/v) precast gel (without Phos-tag) as a control, the 
Tris-Tricine system showed superior results (Figure 5, upper right-hand panel). All the digested peptides that we used were detected in the gel and the presence of small shifts in the mobilities of phosphopeptides (lane: -) from their dephosphorylated counterparts (lane: +) was confirmed. In the Tris-glycine system, on the other hand, each of the digested peptides appeared as a stacked band near gel front (upper left-hand panel). We could not separate theses bands by molecular sieving in the SuperSep Ace precast gel system in conjunction with the Tris-glycine running buffer. Use of the SuperSep Phos-tag precast gel system in conjunction with the Tris-Tricine running buffer permitted the separation and detection of all the digested peptides and it produced more-exaggerated shifts in the mobilities of phosphopeptides (lower right-hand panel). In the Tris-glycine system, however, the AP-treated 14.7 and $11.9 \mathrm{kDa}$ peptides appeared as stacked bands near gel front (lower left-hand panel) in the same manner with the SuperSep Ace precast gel system. These bands were migrated with BPB dye during electrophoresis. Therefore, we could not measure accurately their comparative electrophoretic mobilities in the Tris-glycine system. These results suggest that a combination of SuperSep Phos-tag with Tris-Tricine running buffer provides a higher coverage for a number of phosphoproteins/phosphopeptides and that it generates more-detailed information on the phosphorylation status of proteins/peptides in the low-molecular-mass range.

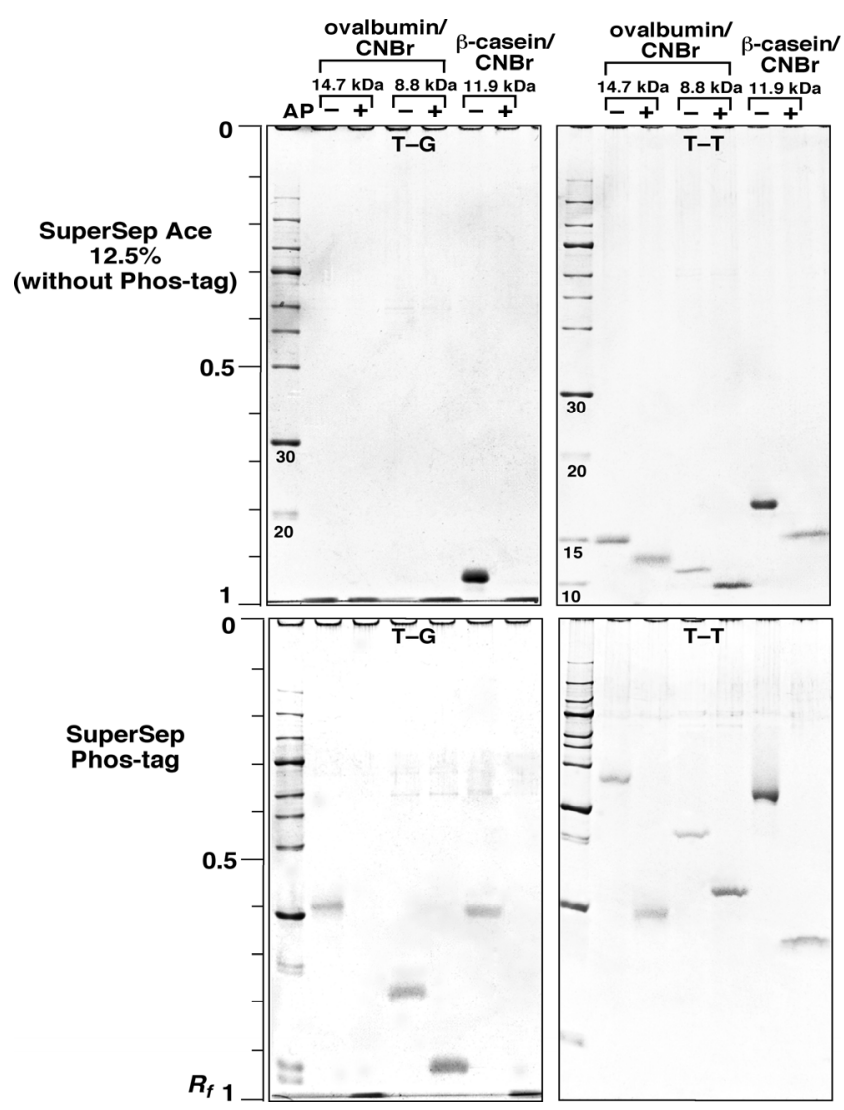

Figure 5. Comparison of mobilities of chemical digested phosphopeptides in the SuperSep Phos-tag system with Tris-glycine (T-G) or Tris-Tricine (T-T) as the running buffer. The phosphopeptides (14.7 and $8.8 \mathrm{kDa}$, lane: - ) obtained by chemical digestion of ovalbumin with $\mathrm{CNBr}$ together with the phosphopeptide (11.9 kDa, lane: -)

obtained by chemical digestion of $\beta$-casein with $\mathrm{CNBr}$, and the corresponding dephosphorylated peptides produced by AP treatment (lane: +) were subjected to electrophoresis on SuperSep Ace (without Phos-tag, upper panels) and SuperSep Phos-tag (lower panels) with Tris-glycine (T-G, left panels) or Tris-Tricine (T-T, right panels) as the running buffer, followed by colloidal CBB staining. Molecular weight markers were applied in the left-most lane in each panel (lane: M). The $R_{f}$ value of 1.0 is defined as the position of the BPB dye.

\section{Conclusion}

Protein phosphorylation, which is one of the most important post-translational modifications, dramatically enhances the diversity of genetically encoded proteins. Many different protein species by phosphorylation site and stoichiometry appear during a number of biological processes. Hyperphosphorylation of a certain protein 
sometimes gives cells or tissues abnormal functions and often introduces pathogenic processes. It has been extremely difficult to pursue the role of variable phosphorylated forms during such processes because current methods treat only crude samples containing the complex proteins. Therefore, the techniques for the separation of the different species of phosphoproteins are very important in phosphoproteomic studies in biological and medical fields. We have demonstrated that it is possible to achieve an improved resolution in the analysis of multiple phosphorylated forms of $\beta$-casein, $\alpha$-casein, and pepsin by the adoption of Tris-Tricine instead of Tris-glycine as an electrophoretic running buffer to the ready-to-use SuperSep Phos-tag system. Furthermore, we have shown that the Tris-Tricine buffer system provides a broader coverage for phosphoproteins/ phosphopeptides in the low-molecular-mass range. Because a downstream procedure, such as Western blotting analysis, is applicable, the technique using the SuperSep Phos-tag precast gel system could assist in mapping low-abundance phosphorylation events. Use of this solid strategy is thus worthy of consideration for a laborsaving, timesaving, and substantial screening in the reliable detection of low-molecular-mass phosphoproteins.

\section{Acknowledgements}

We would like to thank Messrs. Hiroyuki Banba, Akinobu Taniguchi, and Hiroshi Fukui (Wako Pure Chemical Industries, Ltd.) for their kind technical help with SuperSep Phos-tag. This work was supported in part by Grants-in-Aid for Scientific Research (B, 22390006; C, 22590037; C, 24590050) from the Japan Society for the Promotion of Science (JSPS), by a Grant-in Aid for Scientific Research on Innovative Areas (23117522) from the Ministry of Education Culture, Sports, Science, and Technology (MEXT), and by a exploratory research grant (AS232Z01251F) for Adaptable and Seamless Technology Transfer Program through Target-driven R\&D (A-STEP) from the Japan Science and Technology Agency (JST).

\section{References}

Kinoshita, E., \& Kinoshita-Kikuta, E. (2011). Improved Phos-tag SDS-PAGE under neutral pH conditions for advanced protein phosphorylation profiling. Proteomics, 11, 319-323. http://dx.doi.org/10.1002/pmic.201000472

Kinoshita, E., Kinoshita-Kikuta, E., \& Koike, T. (2009). Separation and detection of large phosphoproteins using Phos-tag SDS-PAGE. Nat. Protoc., 4, 1513-1521. http://dx.doi.org/10.1038/nprot.2009.154

Kinoshita, E., Kinoshita-Kikuta, E., \& Koike, T. (2012). Phos-tag SDS-PAGE systems for phosphorylation profiling of proteins with a wide range of molecular masses under neutral $\mathrm{pH}$ conditions. Proteomics, 12 , 192-202. http://dx.doi.org/10.1002/pmic.201100524

Kinoshita, E., Kinoshita-Kikuta, E., Takiyama, K., \& Koike, T. (2006). Phosphate-binding tag, a new tool to $\begin{array}{lllll}\text { visualize phosphorylated proteins. Mol. Cell. Proteomics, 5, 749-757. } & \end{array}$ http://dx.doi.org/10.1074/mcp.T500024-MCP200

Kinoshita, E., Takahashi, M., Takeda, H., Shiro, M., \& Koike, T. (2004). Recognition of phosphate monoester dianion by an alkoxide-bridged dinuclear zinc(II) complex. Dalton Trans., 1189-1193. http://dx.doi.org/10.1039/b400269e

Kinoshita-Kikuta, E., Aoki, Y., Kinoshita, E., \& Koike, T. (2007). Label-free kinase profiling using phosphate affinity polyacrylamide gel electrophoresis, Mol. Cell. Proteomics, 6, 356-366. http://dx.doi.org/10.1074/mcp.T600044-MCP200

Kinoshita-Kikuta, E., Kinoshita, E., \& Koike, T. (2012). Separation and identification of four distinct serine-phosphorylation states of ovalbumin by Phos-tag affinity electrophoresis. Electrophoresis, 33, 849-855. http://dx.doi.org/10.1002/elps.201100518

Laemmli, U. K. (1970). Cleavage of structural proteins during the assembly of the head of bacteriophage T4. Nature, 227, 680-685. http://dx.doi.org/10.1038/227680a0

Schägger, H. (2006). Tricine-SDS-PAGE. Nat. Protoc., 1, 16-22. http://dx.doi.org/10.1038/nprot.2006.4

Schägger, H., \& von Jagow, G. (1987). Tricine-sodium dodecyl sulfate-polyacrylamide gel electrophoresis for the separation of proteins in the range from 1-100 kDa. Anal. Biochem., 166, 368-379. http://dx.doi.org/10.1016/0003-2697(87)90587-2

Steinberg, T. H., Agnew, B. J., Gee, K. R., Leung, W. Y., Goodman, T., Schulenberg, B., ... Patton, W. F. (2003). Global quantitative phosphoprotein analysis using multiplexed proteomics technology. Proteomics, 3, 1128-1144. http://dx.doi.org/10.1002/pmic.200300434 